\title{
Análise comparativa entre métodos ergonômicos analíticos de publicações editoriais impressas e digitais
}

\section{Comparative analysis between analytical ergonomic methods of printed and digital editorial publications}

Marcos Vinícios Schmidt Salvador ${ }^{1}$ Flávio Anthero Nunes Vianna dos Santos ${ }^{2}$ 


\section{Resumo}

Esse estudo tem como objetivo analisar os métodos utilizados por pesquisas que analisem ergonomicamente publicações em suporte digital ou impresso. Por meio de uma revisão bibliográfica, foram selecionados os estudos para a análise. Após a seleçãoᄀ, foram analisados que tipo de método cada estudo utilizou e, então, confrontados. Foram encontradas 9 pesquisas de acordo com os critérios. Descobriu-se que os métodos utilizados são bem distintos, porém houve uma prevalência do questionário pósexperimento. Os protocolos utilizados para análise de publicações em suporte digita utilizam heurísticas específicas que não podem ser transmutados para a análise de publicações impressas. Portanto, a melhor maneira de uma análise comparativa uma análise de experiência de uso.

Palavras-chave: Design Editorial, Expêriencia de Uso, Usabilidade, Dispositivos móveis, Ergonomia.

\section{Abstract}

This study aims to analyze the methods used by surveys that analyzeergonomically publications in digital or printed format. Through a bibliographical review, the studies for the analysis were found. After the selection, the types of method used by each study were analized and then confronted. There were found 9 surveys according to the criteria. It was found that the methods used are quite different but there was a prevalence of the postused to analyze publications in digital format use specific heuristics that can not be transmuted into the analysis of printed publications. Therefore, the best way for a comparative analysis between the two supports is through a use-experience analysis.

Key-words: Ergonomics, Editorial Design User-Experience, Usability, Mobile Devices.
A volatilidade e a conexão social mundial por meio de redes fez com que muitos estudiosos achassem que as publicações impressas fossem perder sua força. O caminho natural parecia ser a extinção desse tipo de publicação. Contudo, estudos publicados pela Deloitte (2018) apontam que o formato impresso ainda é o preferido pelos usuários. 38\% dos leitores de revistas da Australia preferem ler revistas impressas às suas equivalentes digitais. O mesmo estudo afirma que $51 \%$ dos entrevistados preferem consumir notícias de formatos tradicionais do que novas mídias.

A revolução digital possibilitou a publicação em blogs e redes sociais de assuntos de interesse de segmentos específicos de usuários. Assim, assuntos, opiniões e visões de mundo, que antes eram reservados apenas para rodas de conversas off-line, tiveram na internet uma plataforma concreta de difusão global. Através da rede, os usuários puderam se identificar e se reunir para criar publicações sobre assuntos específicos. Pela facilidade de transmissão da informação através da internet, ocorreu uma notória expansão de editores independentes, que cresceram mais que as grandes editoras tradicionais.

Essa mistura de publicações on-line e impressas disponíveis faz com que o consumidor e usuário sejam colocados em um mercado com uma imensa variedade de maneiras de se adquirir uma publicação editorial. Revistas, livros, revistas científicas, repositórios acadêmicos, jornais e uma diversa gama de outros tipos de publicações hoje são disponíveis tanto por meio digital, quanto pelo meio impresso. Portanto, se faz necessária uma verificação da interferência de novas tecnologias na relação entre usuários e novas mídias. E essa análise deve ocorrer através dos requisitos de usabilidade e experiência de uso.

A coexistência entre o suporte impresso e digital é uma constante e apesar de haver alguns altos e baixos no número de publicações impressas perante as digitais, essa coexistência é prevista para existir durante mais algumas décadas. Entretanto, ao que tange a ergonomia, não há muitos estudos sobre esse fenômeno intermodal das publicações editoriais. As análises que existem e podem ser achadas em bases de dados acadêmicas acabam por sempre apontar as falhas ergonômicas em suportes digitais e algumas poucas em suportes físicos. Não foram encontradas análises que apontem para a motivação que leva um usuário escolher entre uma versão ou outra.

A futura aplicação desses métodos analisados pode ser desfrutada futuramente em razão de diferentes objetivos. Desde uma possível escolha mercadológica de se desenvolver uma publicação editorial em determinado suporte em detrimento do outro, como também pode servir de guia para um protocolo de criação de novos sistemas de suporte digital para esses conteúdos. Portanto, este presente artigo tem como objetivos fazer um levantamento de métodos utilizados para análise ergonômica de publicações digitais, fazer também um levantamento voltado para métodos utilizados para análise ergonômica de publicações impressas e comparar esses métodos.

${ }_{1}^{1}$ Mestre em Design, UDESC (contato@marcossalvador.com)

2 Doutor em Engenharia, UFSC (flavio.santos@udesc.br) 


\section{Revisão de Literatura}

A revisão de literatura foi feita por meio de um protocolo. Após definir os objetivos e conceitos dessa revisão, foram definidos os strings de pesquisa necessários para esse levantamento. As bases acadêmicas inicialmente escolhidas para serem utilizadas para a realização dessas pesquisas foram o "Scopus", "WebofScience" e "Scielo". Durante a elaboração e aplicação dos strings foram encontradas grandes dificuldades em refinar os resultados devido à natureza dos termos utilizados. Os termos que definem os objetos de pesquisa das publicações acadêmicas interessantes para esta presente pesquisa, que são publicações editoriais, acabam por não conseguir restringir os resultados. "Magazine", "Book", "Digital Magazine" e "E-magazine" são utilizados nos corpos de textos de praticamente todos os artigos dessas bases, pois esses foram publicados em livros, revistas e etc.

O primeiro string utilizado que fazia referência a publicações digitais teve 194 resultados e a única maneira de restringir esse número foi a verificação manual dos nomes e abstracts. Após essa verificação manual, foram selecionados apenas 3 publicações que correspondiam aos critérios de seleção.

Publicações selecionadas na base de dados "Scopus" após verificação manual dos resultados de pesquisa relacionados ao string (("ergonomics" OR "human factors") and ("method") and ("digital magazine" or "digital book" OR "e-book" OR "e-magazine") and ("usability"))

\begin{tabular}{|c|c|c|c|c|c|}
\hline Nome & Autor(es) & País & $\begin{array}{l}\text { Ano de } \\
\text { publicação }\end{array}$ & $\begin{array}{l}\text { Objeto de } \\
\text { análise }\end{array}$ & Metodologia utilizada \\
\hline \multirow{3}{*}{$\begin{array}{l}\text { A User } \\
\text { Experience } \\
\text { Evaluation } \\
\text { of Amazon } \\
\text { Kindle Mobile } \\
\text { Application }\end{array}$} & \multirow{3}{*}{$\begin{array}{l}\text { Azham } \\
\text { Hussain, } \\
\text { Emmanuel } \\
\text { O.c. } \\
\text { Mkpojiogu, } \\
\text { Ja'afaru } \\
\text { Musa, Salah } \\
\text { Mortada, }\end{array}$} & \multirow[t]{3}{*}{ Malásia } & \multirow[t]{3}{*}{2017} & \multirow[t]{3}{*}{$\begin{array}{l}\text { Aplicativo } \\
\text { Mobile do } \\
\text { Amazon } \\
\text { Kindle }\end{array}$} & $\begin{array}{l}5 \text { tarefas foram dadas para os } \\
\text { participantes executarem no } \\
\text { aplicativo do AmazonKindle, } \\
\text { enquanto um moderador estava } \\
\text { filmando o experimento. }\end{array}$ \\
\hline & & & & & $\begin{array}{l}\text { Foi utilizado o protocolo "think } \\
\text { aloud". }\end{array}$ \\
\hline & & & & & $\begin{array}{l}\text { Foram computados o tempo } \\
\text { de tarefa, frequência de erro e } \\
\text { quantidade de tarefas completas. }\end{array}$ \\
\hline \multirow[t]{2}{*}{$\begin{array}{l}\text { Usability } \\
\text { evaluation } \\
\text { model for } \\
\text { mobile e-book } \\
\text { applications }\end{array}$} & \multirow[t]{2}{*}{$\begin{array}{l}\text { Munya Saleh } \\
\text { Ba Matraf, } \\
\text { Azham } \\
\text { Hussain }\end{array}$} & \multirow[t]{2}{*}{ Malásia } & \multirow[t]{2}{*}{2017} & \multirow[t]{2}{*}{$\begin{array}{l}\text { E-books } \\
\text { mobile }\end{array}$} & $\begin{array}{l}\text { Identificar os requerimentos } \\
\text { para desenvolver os modelos, } \\
\text { ou seja, definir as principais } \\
\text { características e as métricas } \\
\text { associadas. }\end{array}$ \\
\hline & & & & & $\begin{array}{l}\text { Criação de um questionário para } \\
\text { medir a satisfação de uso do } \\
\text { usuário. }\end{array}$ \\
\hline
\end{tabular}

\begin{tabular}{|c|c|c|c|c|c|}
\hline Nome & Autor(es) & País & $\begin{array}{l}\text { Ano de } \\
\text { publicação }\end{array}$ & $\begin{array}{l}\text { Objeto de } \\
\text { análise }\end{array}$ & Metodologia utilizada \\
\hline $\begin{array}{l}\text { A study on } \\
\text { the Usability } \\
\text { of E-books } \\
\text { and APP in } \\
\text { Engineering } \\
\text { Courses: A } \\
\text { Case Study on } \\
\text { Mechanical } \\
\text { Drawing }\end{array}$ & $\begin{array}{l}\text { Min Jou, } \\
\text { Robert D. } \\
\text { Tennyson, } \\
\text { Jingying } \\
\text { Wang, Szu- } \\
\text { Ying Huang }\end{array}$ & $\begin{array}{l}\text { Estados } \\
\text { Unidos/ } \\
\text { Taiwan/ } \\
\text { China }\end{array}$ & 2016 & $\begin{array}{l}\text { Mechanical } \\
\text { drawing } \\
\text { e-books e APP }\end{array}$ & $\begin{array}{l}\text { Technology Acceptance Model } \\
\text { (TAM) }\end{array}$ \\
\hline $\begin{array}{l}\text { Evaluating } \\
\text { user } \\
\text { satisfaction } \\
\text { with } \\
\text { typography } \\
\text { designs via } \\
\text { mining touch } \\
\text { interaction } \\
\text { data in mobile } \\
\text { reading }\end{array}$ & $\begin{array}{l}\text { Junxiang } \\
\text { Wang, } \\
\text { Jianwei Yin, } \\
\text { Shuiguang } \\
\text { Deng, Ying } \\
\text { Li, Calton Pu, } \\
\text { Yan Tang, } \\
\text { Zhiling Luo }\end{array}$ & $\begin{array}{l}\text { Estados } \\
\text { Unidos / } \\
\text { China }\end{array}$ & 2018 & $\begin{array}{l}\text { Tipografia } \\
\text { em Mobile } \\
\text { Learning }\end{array}$ & $\begin{array}{l}\text { Questionário pré-teste, teste, } \\
\text { resposta de um questionário } \\
\text { de compreensão }\end{array}$ \\
\hline $\begin{array}{l}\text { Reading on } \\
\text { Paper and } \\
\text { Screen among } \\
\text { Senior Adults: } \\
\text { Cognitive } \\
\text { Map and } \\
\text { Technophobia }\end{array}$ & $\begin{array}{l}\text { Jinghui } \\
\text { Hou, Yijie } \\
\text { Wu e Erin }\end{array}$ & $\begin{array}{l}\text { Estados } \\
\text { Unidos }\end{array}$ & 2017 & $\begin{array}{l}\text { Leitura de } \\
\text { textos (artigo } \\
\text { científico e } \\
\text { uma história } \\
\text { fictícia) em um } \\
\text { Apple iPad e } \\
\text { Impresso em } \\
\text { papel }\end{array}$ & $\begin{array}{l}\text { Questionário pré-teste, } \\
\text { mensuração de tempo, } \\
\text { resposta de um questionário } \\
\text { de compreensão }\end{array}$ \\
\hline
\end{tabular}

${ }^{1}$ Strings são cadeias de caracteres. Em uma pesquisa bibliográfica, os strings são utilizados para realizar buscas complexas com variabilidade de dados e funções.

Tabela de publicações selecionadas 1. Fonte: Autor

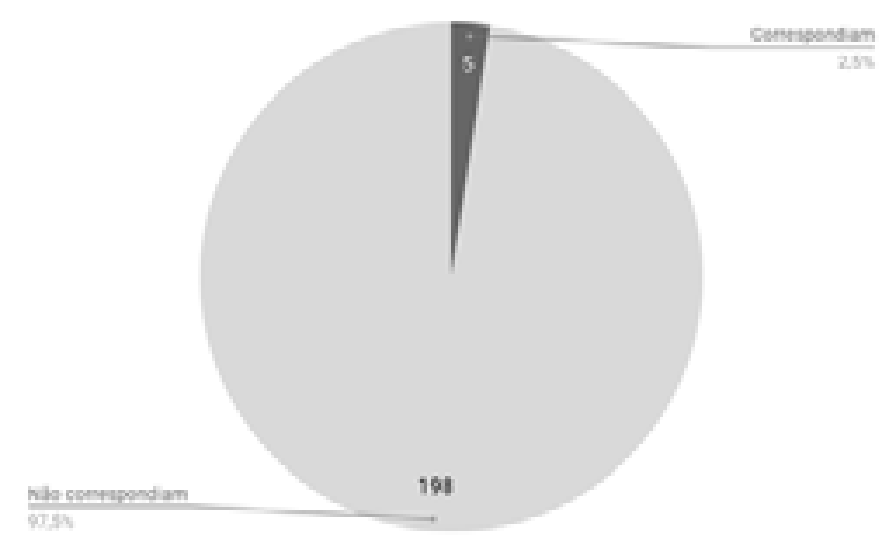

Total de publicações x publicações selecionadas - Gráfico em Pizza. Fonte: Autor 
A partir de então, buscou-se uma pesquisa com termos que poderiam trazer resultados de publicações que analisassem ergonomicamente produtos editoriais impressos, porém nenhum dos resultados nas bases de dados foram relevantes para a pesquisa. Portanto, foi utilizada a base de dados "Google Scholar" para procurar pesquisas nesse sentido. Após essa verificação chegou-se ao seguinte resultado.

Publicações selecionadas na base de dados "Scopus" após verificação manual dos resultados de pesquisa relacionados ao string (("ergonomics" OR "human factors") and ("method") and ("digital magazine" or "digital book" OR "e-book" OR "e-magazine") and ("usability"))

\begin{tabular}{|c|c|c|c|c|c|}
\hline Nome & Autor(es) & País & $\begin{array}{l}\text { Ano de } \\
\text { publicação }\end{array}$ & $\begin{array}{l}\text { Objeto de } \\
\text { análise }\end{array}$ & Metodologia utilizada \\
\hline $\begin{array}{l}\text { Características } \\
\text { da interface } \\
\text { dos sites de } \\
\text { notícias: um } \\
\text { estudo do } \\
\text { design e da } \\
\text { usabilidade } \\
\text { de jornais } \\
\text { impressos e } \\
\text { eletrônicos }\end{array}$ & $\begin{array}{l}\text { Danielle de } \\
\text { Almeida } \\
\text { Pacheco } \\
\text { Thomaz }\end{array}$ & Brasil & 2009 & $\begin{array}{l}\text { Jornal o Estado de } \\
\text { S. Paulo e Jornal } \\
\text { Folha de São Paulo }\end{array}$ & $\begin{array}{l}\text { Questionaire for User } \\
\text { Interaction Satisfaction } \\
\text { (Questionário para a } \\
\text { Satisfação da Interação } \\
\text { do Usuário, QUIS) }\end{array}$ \\
\hline $\begin{array}{l}\text { Ver, } \\
\text { Compreender } \\
\text { e Aprender: } \\
\text { Estudo } \\
\text { Comparado } \\
\text { com Guias de } \\
\text { Uso de Apps } \\
\text { Editoriais para } \\
\text { Dispositivos } \\
\text { Móveis }\end{array}$ & $\begin{array}{l}\text { Ana C. } \\
\text { Dalagnoli, } \\
\text { Flávio A. N. } \\
\text { V. Santos }\end{array}$ & Brasil & 2016 & $\begin{array}{l}\text { Guia de instruções } \\
\text { para a utilização } \\
\text { de aplicativos para } \\
\text { iPad das revistas de } \\
\text { decoração e design } \\
\text { de interiores Casa } \\
\text { Claudia (Editora } \\
\text { Abrill) e Casa e } \\
\text { Jardim (Editora } \\
\text { Globo) }\end{array}$ & $\begin{array}{l}\text { Questionário Pré- } \\
\text { experimento (Pré- } \\
\text { teste). } \\
\text { Testes de Usabilidade } \\
\text { com observação de } \\
\text { moderador } \\
\text { e Think-aloud. }\end{array}$ \\
\hline $\begin{array}{l}\text { Avaliação da } \\
\text { revista Info } \\
\text { Exame a partir } \\
\text { de heurísticas } \\
\text { para tablets }\end{array}$ & $\begin{array}{l}\text { Diego } \\
\text { Borges } \\
\text { da Silva, } \\
\text { Berenice S. } \\
\text { Gonçalves }\end{array}$ & Brasil & 2015 & $\begin{array}{l}\text { Aplicativo para } \\
\text { Ipad da revista } \\
\text { InfoExame }\end{array}$ & $\begin{array}{l}\text { Análise das heurísticas } \\
\text { com foco em } \\
\text { publicações para } \\
\text { tablets, proposta } \\
\text { por Heikkilä (2013): } \\
\text { acessibilidade, } \\
\text { usabilidade e } \\
\text { experiência do usuário }\end{array}$ \\
\hline $\begin{array}{l}\text { Eye-Movement } \\
\text { Patterns in } \\
\text { Disabled } \\
\text { Readers at } \\
\text { Two Age } \\
\text { Levels: A Test } \\
\text { of Bakker's } \\
\text { Balance Model }\end{array}$ & $\begin{array}{l}\text { Jacques } \\
\text { Donders, } \\
\text { Harry van } \\
\text { der Vlugt. }\end{array}$ & Brasil & 2004 & $\begin{array}{l}\text { Protótipos de } \\
\text { páginas de jornal }\end{array}$ & Eye-Tracking \\
\hline
\end{tabular}

Tabela de publicações selecionadas 2. Fonte: Autor

\section{Breve resumo dos métodos análiticos utilizados pelos estudos encontrados}

O artigo "A User Experience Evaluation of Amazon Kindle Mobile Application" (2017) faz uma análise ergonômica da usabilidade do aplicativo mobile do Amazon Kindle $^{\mathrm{TM}}$. O experimento foi realizado na biblioteca e na cafeteria de uma residência estudantil da Universiti Utara Malaysia. Os usuários ficaram livre para realizar as tarefas designadas tanto em pé quanto sentados. Haviam dois moderadores, um era encarregado de orientar para quais tarefas deveriam ser executadas, e o segundo era responsável por captar em vídeo, por meio da câmera de um smartphone, as sessões de teste. Após as tarefas serem apresentadas aos participantes do experimento, não era imposta nenhuma restrição sobre a forma de agir. Além de filmar a tela e os movimentos que os participantes realizavam no aplicativo instalado nos smartphones Android, o tempo de cada tarefa também era cronometrado. Um total de 5 tarefas foram apresentadas para os 15 estudantes que participaram do experimento. Além disso, os participantes eram encorajados a falar em voz alta o que estavam pensando $e$ executando naquele momento, método conhecido como Think aloud. Imediatamente após o final do teste, cada participante respondia a um questionário para responder questões que mensuram cada fator de interesse do teste de usabilidade. Finalizando, os dados quantitativos de tempo de tarefa, frequência de erro e taxa de sucesso foram computados. Esses resultados foram analisados utilizando o software SPSS (versão 23)

A proposta da publicação "Usability Evaluation Model for Mobile E-Book Applications" (2017) é diferente, pois esta se propõe a identificar quais elementos na avaliação de usabilidade são mais significativos na relação com a satisfação do usuário. $O$ estudo fez uma revisão de literatura para definir quais heurísticas seriam utilizadas para a avaliação de usabilidade de um determinado aplicativo de e-book. As heurísticas categorizadas foram: Leiturabilidade, efetividade, acessibilidade, eficiência e navegação. 30 participantes participaram do experimento utilizando apps leitores de e-books. O tempo para completar as tarefas dadas foram cronometrados. Após o teste de realização de tarefas, foi efetuado um questionário e esses dados foram tabulados no software SPSS (versão 23). Os dados foram utilizados para analisar a hipótese de relação entre a satisfação do usuário e as heurísticas levantadas. O resultado demonstrou que a satisfação de usuários de e-books era significantemente influenciada pelas cinco características que foram apontadas. $O$ estudo também demonstrou que 3 dessas heurísticas possuíam uma maior relação do que as outras com o uso de leitores de e-books, são elas: Navegação, efetividade e acessibilidade.

"A study on the Usability of E-books and APP in Engineering Courses: A Case Study on Mechanical Drawing" avalia a usabilidade de e-books e apps em cursos de engenharias. Para a finalidade do estudo aqui sendo realizado, será apenas indicado os aspectos da pesquisa desse artigo que sejam referidos à análise dos e-books, portanto, estamos descartando a pesquisa feita baseada na usabilidade dos apps. Utilizando o Modelo de Aceitação de Tecnologia (TAM) proposto por Davis (1986) em sua tese de doutorado, foram analisadas as 3 fases de percepção, intenção e uso. Cada fase incluiu vários aspectos ligados a usabilidade de e-books. Após pesquisa bibliográfica e sanção de profissionais da área chegou-se à 11 heurísticas de usabilidade: conveniência, compatibilidade, utilidade percebida, gerenciamento de tempo, autoavaliação, ajuste da tarefa tecnológica, efetividade da tarefa tecnológica, expectativa de desempenho, 
estética, satisfação do usuário e intenção de uso. Antes da implementação oficial do questionário de avaliação de usabilidade, ele foi testado durante três anos consecutivos. Após a aplicação do questionário, os dados foram quantificados baseados na experiência de aprendizado de acordo com as diferentes experiências (e-books ou apps).

O artigo "Evaluating User Satisfaction with Typography Designs via Mining Touch Interaction Data in Mobile Reading" apresenta um experimento para analisar a satisfação do usuário com os designs da tipografia na leitura feita em suportes mobile. Para o teste foram realizados questionários pré-teste nos 52 participantes e depois solicitado que lessem 5 diferentes textos em tipografias e tamanhos diferentes. Após a leitura dos textos, os usuários responderam questões de múltipla escolha sobre os textos lidos. Imediatamente após, foram dadas notas qualitativas de 1 a 5 sobre a percepção do usuário em relação aos textos.

No estudo "Reading on Paper and Screen among Senior Adults: Cognitive Map and Technophobia", foi realizado um experimento com pessoas acima de 50 anos, onde foi feito um questionário pré-experimento. Após esse procedimento, os participantes foram expostos a leitura de dois textos (um artigo científico e uma história de ficção), um em suporte digital (Apple iPad) e outro em suporte impresso. Ambos os textos possuiram mesmo tamanho, tamanho de fonte e diagramação. Foram medidos os tempos de leitura e após o teste foi aplicado um teste de compreensão dos textos lidos.

No artigo "Avaliação da Revista Info Exame a partir de Heurísticas para Tablets", a avaliação das heurísticas foi feita por dois profissionais com conhecimento em usabilidade. Essa avaliação foi apresentada por meio de um relatório baseado nos problemas encontrados na edição número 352. Foram utilizados os três pilares sugeridos por Heikkilä (2013): acessibilidade, usabilidade e experiência do usuário como guia para essa análise executada pelos profissionais.

No estudo "Ver, Compreender e Aprender: Estudo Comparado com Guias de Uso de Apps Editoriais para Dispositivos Móveis" feito por Dalagnoli e Santos (2016), foi comparado o guia de uso dos aplicativos das revistas "Casa Claudia" e "Casa e Jardim" para o dispositivo "iPad". Foram selecionados os participantes da pesquisa baseando-se em uma análise do público consumidor dessas revistas. Realizou-se um pré-teste antes do experimento. $O$ experimento em si baseou-se na execução de tarefas apresentadas pela moderadora aos participantes com o apoio do guia de uso disponibilizado pelas editoras. Os participantes eram encorajados a verbalizar em voz alta os pensamentos que elas tinham, método conhecido como Think Aloud. Após o experimento, os dados foram tabulados e analisados.

Na dissertação "Características da Interface dos Sites de Notícias: Um Estudo do Design e da Usabilidade de Jornais Impressos e Eletrônicos" (2009) é utilizada as diretrizes propostas por Shneiderman (2005), com o Questionaire for User Interaction, para a criação das questões relativas aos questionários que foram aplicados na pesquisa. Esses questionários foram utilizados para analisar os jornais impressos e digitais "O Estado de S. Paulo" e "Folha de S. Paulo". Como o estudo é de 2009, para a presente pesquisa, foram desconsiderados os estudos feitos relacionados aos websites. Além da evolução nos elementos tecnológicos ocorrida da época da dissertação até o momento deste presente estudo, a pesquisa estudou apenas a página principal dos websites. Os questionários possuíam 12 questões com um total de 30 itens. Foram analisadosaspectos de design, ergonomia cognitiva e, mais especificamente, usabilidade. Anteriormente a aplicação dos questionários foi feito um pré-teste a fim de verificar dificuldades na interpretação das perguntas e das respostas. Após a análise oficial dos resultados os dados foram tabulados e aplicou-se uma análise estatística para obtenção das médias e do desvio padrão. O teste utilizado foi o Wilcoxon, utilizando o software Statistica 7.0.

O estudo mais antigo analisado por esse artigo foi a dissertação de mestrado "Eye movement patterns and newspaper design factors - An experimental approach" de Holmberg (2004). Foram utilizados 12 participantes com visão normal que utilizaram um rastreador de olhar iView da SensoMotoric Instruments (SMI). Apesar da visão ser biocular, somente os movimentos do olho direito foram monitorados. Esses participantes foram estimulados a ler dois diferentes protótipos de jornais impressos com diagramações diferentes, porém com a mesma capa e contra-capa. Após o experimento foram analisadas as imagens com o mapa que foi gerado por meio do rastreador.

\section{Estudo comparativo entre os métodos encontradas}

Referindo-se a experiência de uso, a maior parte dos estudos encontrados utilizase de questionários pós-experimentos. As heurísticas utilizadas para a elaboração desses questionários variam de acordo com os pesquisadores. Os questionários são apresentados como uma possibilidade dentro do método "Entrevista" sugerido por Young e Stanton (2005) no "Handbook of Human Factors and Ergonomics Methods". Os questionários não são administrados pelo pesquisador e podem ser completados em forma física ou digital pelo próprio participante do estudo. Essa maneira de diagnosticar acaba por ser útil devido a possibilidade de haver uma grande amostra de participantes. Entretanto, a realização de questionários pós-experimento acaba por limitar a flexibilidade das questões e, consequentemente, das respostas e a impossibilidade de encontrar problemas ergonômicos que por ventura não tenham sido preliminarmente tidos como possíveis pelo pesquisador.

Outro protocolo utilizado em muitos dos estudos encontrados foi o Think Aloud. O Think Aloud é um protocolo organizado em 1993 por Lewis e Rieman e conceitualmente é muito simples. É pedido para os usuários cumprirem uma tarefa, mas também é pedido para que eles conversem com o pesquisador enquanto tentam cumprir aquela meta. O pesquisador pede para os participantes que eles digam em voz alta o que eles estão pensando: $O$ que eles estão tentando fazer, questões que tangenciam o cumprimento da tarefa, entre outros. O pesquisador pode gravar o que está sendo dito, ou apenas tomar notas. É também encorajado que o pesquisador converse com o participante enquanto este exerce sua tarefa. É notado que quando isso não acontece, os participantes não desempenham um bom ritmo de comentários. Porém, o pesquisador não deve falar diretamente sobre a tarefa que eles estão realizando, mas sim exercer incentivos como "continue falando" ou "diga-me o que você está pensando" durante a execução do teste.

Foi percebido com esta pesquisa que não é fácil encontrar estudos que fazem uma análise ergonômica de publicações impressas. Portanto, as duas pesquisas encontradas neste presente estudo possuem métodos e finalidades distintos. Enquanto um estudo 
utiliza um protocolo de questionário para analisar o design, ergonomia cognitiva e usabilidade do objeto, o outro utiliza uma ferramenta tecnológica de rastreamento ocular para gerar mapas de visão sobre o objeto de análise. Enquanto o primeiro utiliza de ferramentas mais acessíveis, o segundo utiliza uma ferramenta cara e muitas vezes indisponível para o pesquisador. $O$ estudo que utiliza o protocolo de questionário pré-teste, teste e questionário pós-teste se aproxima mais metodologicamente dos estudos feitos com publicações em suporte digital. Portanto, caso em algum estudo futuro se veja necessário fazer um teste comparativo entre o suporte físico e digital, o protocolo mais indicado e acessível seria esse. O questionário pré-teste deverá aborda aspectos sócio-econômicos, bem como, variáveis que possam influenciar na relação entre o usuário e o objeto do estudo. Os testes realizados deverão ser testes que unam medidas quantitativas com dados qualitativos, pois a comparação apenas quantitativa entre duas mídias de suporte diferente pode ter resultados imprecisos. Portanto a realização de tarefas semelhantes nos dois suportes com métodos em que o usuário possa se expressar durante a realização (o think-aloud, por exemplo) é aconselhado. Além disso, é de suma importância que o ergonomista acompanhe o teste e faça anotações, principalmente para determinar quais caminhos cognitivos são adotados nos diferentes suportes. O questionário pós-teste deverá apresentar questões relativas a experiência do uso e de como o usuário se sentiu ao realizar o teste. A aplicação de um questionário estruturado é recomendada para a verificação comparativa estatística posterior. Todavia, uma entrevista posterior a realização do questionário estruturado se faz necessária. Aspectos importantes relacionados a afetividade podem ser descobertos por meio dela.

Algumas análises de publicações digitais encontradas utiliza como um dos métodos a análise heurística, a maior parte desses protocolos são baseados na proposta de Nielsen (1994). Nielsen propõe dez heurísticas para serem avaliadas para uma boa usabilidade. Alguns desses estudos utilizam-se de protocolos já existentes que surgiram a partir da proposta de Nielsen, porém com ajustes. Outro ainda faz uma reinterpretação dessas heurísticas, utilizando apenas aquelas que se mostram de maior relevância para o estudo em si. As heurísticas propostas por Nielsen são 10: Visibilidade de qual estado estamos no sistema; Correspondência entre o sistema e o mundo real Liberdade de controle fácil pro usuário; Consistência e padrões; Prevenção de erros; Reconhecimento em vez de memorização; Flexibilidade e eficiência de uso; Estética e design minimalista; Ajude os usuários a reconhecerem, diagnosticarem e recuperarem se de erros; Ajuda e documentação. Porém ao transmutar essa avaliação heurística para fazer uma avaliação de uma publicação em suporte impresso muitas dificuldades podem ser encontradas pelo fato de as heurísticas de Nielsen serem normalmente aplicadas às avaliações de usabilidade homem-máquina, principalmente na área de ciências da computação.

Portanto, para haver a possibilidade de um teste comparativo entre o suporte digital e o suporte impresso, a análise precisa ter como base algo mais amplo conceitualmente. $O$ estudo encontrado que realiza esse comparativo, utiliza-se de um questionário pré-teste, e depois realiza um teste de compreensão dos textos em ambos os suportes. A mensuração é para descobrir algo bem específico: se a tipografia em diferentes suportes exerce influência sobre a compreensão dos textos pelos indivíduos. Outra maneira viável de comparar ergonomicamente publicações em suportes digitais e impressos seria a análise da experiência do usuário de publicações onde outros fatores podem ser levados em consideração. A experiência do usuário é considerada uma expansão do conceito de usabilidade. A norma ISO 9241-210 (2008) define experiência do usuário como: "as percepções de uma pessoa e as respostas que resultam do uso e/ ou do uso antecipado de um produto, sistema ou serviço". A avaliação da experiência do usuário permite ainda que aspectos subjetivos que influenciam na relação usuáriopublicação sejam mensurados. Reiss (2014) define experiência do usuário como a percepção deixada na mente de alguém após uma série de interações entre pessoas, dispositivos e eventos - ou qualquer combinação destes. Essas interações podem ser ativas como clicar um botão em um e-book, comprar uma revista em uma banca; ou ainda passivas como a satisfação de ver algo que você gosta muito em uma revista de grande circulação, que acaba desencadeando a liberação de substâncias químicas de recompensa no cérebro.

\section{Bibliografia}

BARON, Naomi. Words onscreen. 1. ed. Washington: Oxford University Press, USA, 2015.

DA SILVA, Diego Borges; GONÇALVES, Berenice S. Avaliação da revista Info Exame a partir de heurísticas para tablets. SIGRADI. 2015.

DALAGNOLI, Ana C; SANTOS, Flávio A N V. Ver, Compreender e Aprender: Estudo Comparado com Guias de Uso de Apps Editoriais para Dispositivos Móveis. Design \& Tecnologia 12. 2016.

DAVIS, Fred D. Technology Acceptance Model for Empirically Testing New End-User Information Systems: Theory and Results. Tese de doutorado. MIT Sloan School of Management: Cambridge, MA. 1986

DELOITTE Touche Tohmatsu Australia. Media Consumer Survey 2018 - Australian media and digital entertainment preferences. Sydney: [s.n.], 2018. Disponível em: $<$

http://images.content.deloitte.com.au/Web/DELOITTEAUSTRALIA/\%7Bcdafce4f-483e49b4-8f00-cbb947586250\%7D_20180914-tel-inbound-media-consumer-survey-2018report.pdf?elqTrackld=7d262a84bd414084877f28acba8f5876\&elqaid=227\&elqat $=2$

>. Acesso em: 19 abr. 2019.

DONDERS, J.; VAN DER VLUGT, H. Eye-Movement Patterns in Disabled Readers at Two Age Levels: A Test of Bakker's Balance Model. Journal of Clinical Neuropsychology, 6(3), 241-256. 1984

GRUSZYNSKI, Ana C; GOLIN, Cida; LUCHESE, Alexandre F. Desafios para a comunicação da ciência: um estudo sobre os periódicos científicos impressos e eletrônicos da UFRGS.

HFD, v.9, n 17, p. 3-14, junho 2020 
XXX Congresso Brasileiro de Ciências Da Comunicação, 1-15. 2006

HEIKKILÄ, Harri. Towards tablet publication heuristics. Improving accessability, usability and user experience with new expert Evaluation. 2013. Disponível em: http://www. mediaconceptsrg.net/wp-content/uploads/2011/05/D1.2.1.1_eReading_Towards-tabletpublication-heuristics.pdf. Acesso 8 jun. 2018.

HUSSAIN, Azham; MKPOJIOGU, Emmanuel O C; MUSA, Ja'afuru; MORTADA, Salah. A user experience evaluation of Amazon Kindle mobile application. The 2nd International Conference on Applied Science and Technology. 2017

JOU, Min; TENNYSON, Robert D; WANG, Jingying; HUANG, Szu-Ying. A study on the usability of E-books and APP in engineering courses: A case study on mechanical drawing. Computers and Education, 92-93, 181-193. 2016

LEWIS, Clayton; RIEMAN, John. Task-centered user interface design. Boulder, Colorado: University of Colorado, Boulder, Dept. of Computer Science. 1993

MATRAF, Munya S B; HUSSAIN, Azham. Usability evaluation model for mobile e-book applications. The 2nd International Conference on Applied Science and Technology. 2017

NIELSEN, Jakob. Heuristic evaluation. In Nielsen, J., and Mack, R.L. (Eds.), Usability Inspection Methods. New York, NY: John Wiley \& Sons. 1994

HOU, Jinghui; WU, Yijie; HARRELL, Erin. Reading on paper and screen among senior adults: Cognitive map and technophobia. Frontiers in Psychology, vol. 8. 2018

SHNEIDERMAN, Ben. Designing the user interface: strategies for effective humamcomputer interaction. Boston: Pearson, 2005.

STANTON, Neville; YOUNG, Mark. Applying Interviews to Usability Assessment. In: STANTON, Neville. Handbook of Human Factors and Ergonomics Methods. Boca Raton: CRC Press. 2005

THOMAZ, Danielle A P. Características Da Interface Dos Sites De Notícias: Um Estudo Do Design E Da Usabilidade De Jornais Impressos E Eletrônicos. UNESP Universidade Estadual Paulista Júlio de Mesquita Filho - Programa de Pós-Graduação Em Design, Da Faculdade de Arquitetura, Artes E Comunicação, 81. 2009.

WANG, Junxiang; YIN, Jianwei; DENG, Shuiguang; LI, Ying; PU, Calton; TANG, Yan; LUO, Zhiling. Evaluating user satisfaction with typography designs via mining touch interaction data in mobile reading. Conference on Human Factors in Computing Systems. Proceedings: 2018. 